\title{
The Role of Atopy in the Choroidal Profile of Keratoconus Patients
}

\author{
Ana Filipa Moleiro (iD) ${ }^{1,2}$ \\ Ana Francisca Aires ${ }^{3}$ \\ Hélio Alves ${ }^{4}$ \\ João Viana Pinto (iD ${ }^{5}$ \\ Ângela Carneiro ${ }^{1,2}$ \\ Fernando Falcão-Reis ${ }^{1,2}$ \\ Luís Figueira ${ }^{1,4}$ \\ João Pinheiro-Costa ${ }^{1,4}$ \\ 'Department of Ophthalmology, Centro \\ Hospitalar Universitário São João, Porto, \\ Portugal; ${ }^{2}$ Department of Surgery and \\ Physiology, Faculty of Medicine, \\ University of Porto, Porto, Portugal; \\ ${ }^{3}$ Faculty of Medicine, University of Porto, \\ Porto, Portugal; ${ }^{4}$ Department of \\ Biomedicine, Faculty of Medicine, \\ University of Porto, Porto, Portugal; \\ ${ }^{5}$ Department of Otorhinolaryngology, \\ Centro Hospitalar Universitário São João, \\ Porto, Portugal
}

Purpose: Although classically classified as a non-inflammatory condition, an inflammatory basis for keratoconus (KC) appears to be a growing evidence. Recently, it has been shown that $\mathrm{KC}$ patients have an increased choroidal thickness (CT). Among inflammatory disorders, atopy has been associated with $\mathrm{KC}$ development; therefore, the aim of this study was to evaluate if the increased $\mathrm{CT}$ in patients with $\mathrm{KC}$ is related to atopy.

Methods: This is an analytical cross-sectional study of patients with KC. Patients were classified as atopic and non-atopic according to their atopy history (allergic rhinoconjunctivitis (AR), asthma (AA) and/or atopic dermatitis (AD)) and were also classified based on their eye rubbing habits. Choroidal profile of all subjects was evaluated using a Spectralis optical coherence tomography (OCT) device with enhanced depth imaging (EDI) mode. CT was measured and compared between groups at the center of the fovea and at $500 \mu \mathrm{m}$ intervals along a horizontal section. A multivariable analysis, adjusted for sex, age, spherical equivalent, history of medication and atopy, was performed to assess the influence of atopy in CT.

Results: Of the 80 patients included, 51 were atopic and 29 non-atopic. Atopic patients showed a thicker choroid in every measured location than the non-atopic patients (mean subfoveal CT $391.53 \mu \mathrm{m}$ vs $351.17 \mu \mathrm{m}$, respectively), although the differences were not statistically different. The multivariable analysis revealed that being atopic makes the choroid statistically thicker, on average, $55.14 \mu \mathrm{m}$, when compared to non-atopic patients $(p=0.043)$. Furthermore, patients who are frequent eye rubbers have significantly thicker choroids than non-rubbers $(\mathrm{p}=0.004)$.

Conclusion: Although some results do not reach statistical significance, atopic KC patients seem to have thicker choroids compared with non-atopic KC patients, suggesting a possible role for atopy in the choroidal profile of KC. This constitutes a completely new sight in this field of research that needs further investigation.

Keywords: atopy, choroid, choroidal thickness, cornea, keratoconus

\section{Introduction}

Keratoconus $(\mathrm{KC})$ has been classically classified as a non-inflammatory condition since there was no evidence of inflammatory cell infiltration or neovascularization in the cornea. ${ }^{1,2}$ However, the paradigm has been changed in the last years. Several studies evidenced the imbalance between inflammatory cytokines, proteases, and proteases inhibitors, as well as free radicals and oxidants, with a pivotal role in $\mathrm{KC}$ pathogenesis. $^{3-9}$

The plausibility of inflammation as a potential cause of $\mathrm{KC}$ is reinforced since atopy and ocular surface inflammation have been associated with a higher prevalence of $\mathrm{KC} .^{10}$ 
Indeed, eye rubbing habits and atopy are among the most important risk factors for KC. ${ }^{11}$ According to a recent meta-analysis, the odds ratio of developing $\mathrm{KC}$ was 3 times higher in subjects who had abnormal eye rubbing habits on a daily basis compared with those who did not have this habit. ${ }^{11,12}$ Atopy has been associated with $\mathrm{KC}$ development, which may be in part attributed to the increased potential to eye rubbing habits. Although some controversy about its role as a risk factor per se, some large studies have shown a significant difference in the frequency of atopy in KC. Furthermore, asthma, allergy, and eczema were all identified as effective risk factors for $\mathrm{KC} .{ }^{11}$

An increased Choroidal Thickness (CT) in KC patients has recently been described. ${ }^{13-15}$ However, the exact changes taking place in the choroid itself are still unclear. Choroid has been implicated in the pathogenesis of many inflammatory disorders of the eye $\mathrm{e}^{16-18}$ and seems to be subclinically involved in some systemic inflammatory diseases without ophthalmologic manifestations. ${ }^{19}$

The more recent theories about the pathophysiology of $\mathrm{KC}$ hint to a possible inflammatory component, which might explain the increased CT profile evidenced in this population. $^{1,13,14}$

Our group have previously reported a thicker macular choroid in KC eyes compared to age-matched controls. ${ }^{13}$ However, as atopic conditions were not an exclusion criterion, and risk factors data were analyzed from medical records, the role of atopic disease in CT remained unclear. Bearing in mind that atopy is very common in patients with $\mathrm{KC}$, and that the mechanisms underlying allergic rhinoconjunctivitis and atopic dermatitis are closely related to eye rubbing habits, atopy may play a role in both the development of $\mathrm{KC}$ and the increase of CT.

Based on this evidence, our goal was to find if atopy has a role in the choroidal profile of $\mathrm{KC}$ patients.

\section{Methods}

\section{Patient Selection and Data}

We conducted an analytical cross-sectional study to assess the atopy history and the eye rubbing habits of $\mathrm{KC}$ patients. Eighty patients aged 14 to 30 years old, followed in our Cornea Department, were identified and consecutively included in the study, between September and December 2019.

The study was conducted at Centro Hospitalar Universitário São João (CHUSJ), Porto, Portugal, and the study protocol was approved by the Ethics Committee of Health of CHUSJ, following the tenets of the Declaration of Helsinki (2008; 2013). Informed consent was obtained from all participants and/or their legal guardians after full explanation of the procedures.

A combination of a questionnaire and allergology medical record consultation was performed to collect atopy history. The following features were collected: eye's rubbing habits, history of atopic diseases and anti-inflammatory therapy history. Atopy was defined as the presence of at least one of the following three conditions: AR, AA and AD. Eye rubbing habits were classified as: none (never rub their eyes), rarely (less than one time per week), sometimes (between one and several times per week) or frequently (everyday). Considering anti-inflammatory therapy history, the following parameters were screened: systemic antiinflammatory use, ocular anti-inflammatory use, topical antiinflammatory use (including nasal sprays, inhalators and ointments). As anti-inflammatory therapy, the following medications were considered: antihistaminic, non-steroidal anti-inflammatory or corticosteroid drugs. Regarding all these medications, it was recorded if medication was currently or in the past in use and when it was used for the last time. The use of frequent rescue medication, including nasal and inhalator drugs, as beta-agonists, was also recorded.

All patients performed a complete ophthalmic examination to exclude other ocular pathologies (best-corrected Snellen visual acuity (BCVA), biomicroscopic examination, intraocular pressure and fundus examination). The morphological characterization of the cornea was performed using Pentacam HR (OCULUS Optikgeräte $\mathrm{GmbH}$, Wetzlar, Germany).

The following exclusion criteria were considered: existence of other ocular pathology than $\mathrm{KC}$; known systemic diseases rather than allergic conditions; existence of active systemic or ocular inflammation; anti-inflammatory therapy (systemic, ocular or topical) in the month prior to the study; other ocular surgeries besides intracorneal ring segments or crosslinking procedures (performed at least 6 months prior to the scan). Inclusion and exclusion criteria are presented in Table 1.

Patients that met inclusion criteria performed an SDOCT with enhanced depth imaging (EDI) technology to evaluate choroidal profile. All OCT scans were acquired by a trained technician in normal follow-up assessments, at morning and without contact lenses in all patients.

\section{Choroidal Imaging Protocol}

The patients underwent EDI SD-OCT using the Spectralis Heidelberg apparatus (Heidelberg Engineering Inc, 
Table I Inclusion and Exclusion Criteria

\begin{tabular}{|l|}
\hline Inclusion Criteria \\
\hline Patients diagnosed with KC (any stage) \\
Age between I4 and 30 years old \\
Continuous follow-up at the Corneal Department of CHUS \\
\hline Exclusion Criteria \\
\hline $\begin{array}{l}\text { Existence of any ocular pathology other than KC } \\
\text { Eyes with other ocular surgeries besides intracorneal ring segments or } \\
\text { crosslinking procedures (performed at least } 6 \text { months prior to the study) } \\
\text { Current treatment with any anti-inflammatory medication (systemic, } \\
\text { ocular or topical) in the last month prior to the inclusion; only } \\
\text { artificial tears were accepted } \\
\text { Any other systemic diseases rather than atopic conditions (AR, AD } \\
\text { and/or AA) } \\
\text { Eyes with poor quality SD-OCT scans, where it is difficult to } \\
\text { differentiate clearly the choroidal-scleral junction }\end{array}$ \\
\hline
\end{tabular}

Note: Inclusion and exclusion criteria used in the study.

Abbreviations: KC, keratoconus; SD-OCT, spectral-domain optical coherence tomography; CHUSJ, Centro Hospitalar e Universitário de São João; AR, allergic rhinitis; $A D$, atopic dermatitis; $A A$, allergic asthma.

Heidelberg, Germany). The choroidal imaging protocol was applied according to the previously published in 2019 by Pinheiro-Costa et al. ${ }^{13}$ The SD-OCT scans were single $30^{\circ} \mathrm{B}$-scans centered on the fovea using the EDI function averaged 100 times. CT was measured from the outer edge of the hyperreflective line, corresponding to the retinal pigment epithelium, to the choroidal-scleral junction (Figure 1). These measurements were taken at the subfoveal choroid and at $500 \mu \mathrm{m}$ intervals from the fovea: temporal $500 \mu \mathrm{m}$ (T500), $1000 \mu \mathrm{m}$ (T1000), 1500 $\mu \mathrm{m}(\mathrm{T} 1500)$ and nasal $500 \mu \mathrm{m}$ (N500), $1000 \mu \mathrm{m}$ (N1000) and $1500 \mu \mathrm{m}$ (N1500). Only high-quality images were considered and all the images with a poor choroidalscleral junction visibility were excluded. CT measurements were performed and confirmed manually by two masked independent observers (JVP and JPC; interobserver intraclass correlation $0.975,95 \%$ confidence interval 0.965-0.982). An illustrative image of the choroidal measurements is presented in Figure 1.

\section{Statistical Evaluation}

Only one eye of each patient was used for statistical analysis (the right eye was chosen when both eyes met the inclusion criteria). The Kolmogorov-Smirnov test and normal probability plots were used to confirm the normal distribution of the data. Parametric or non-parametric tests were used for continuous variables comparison between the atopic and non-atopic group, according to the normality of the data. Chi-square or Fisher's exact tests were performed for categorical variables comparison. Multivariable linear regression analysis (adjusted to covariates age, sex, spherical equivalent, history of medication and atopy) was performed to identify the potential variables associated with subfoveal CT. Statistical significance for all the analyses was set at a $\mathrm{p}$ value $<0.05$. Statistical analysis was conducted using SPSS statistical software package version 24 (SPSS Inc., Chicago, IL, USA).

\section{Results}

A total of 80 eyes of 80 patients met the inclusion criteria and were analyzed, from which 61 were male and 19 female, with a mean age of $24.5 \pm 4.4$ years. All the included patients were

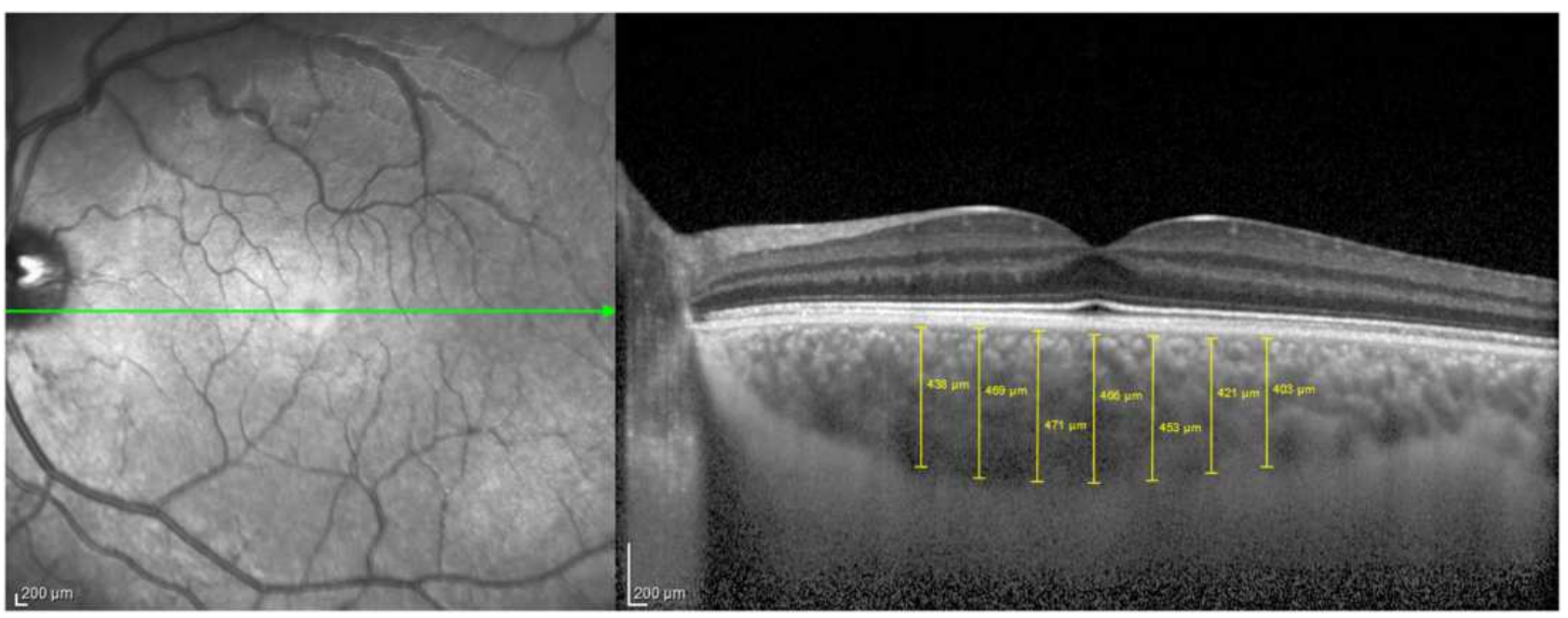

Figure I Schematic representation of choroidal thickness measurement. Choroidal Thickness was measured from the outer edge of the hyperreflective line, corresponding to the retinal pigment epithelium, to the choroidal-scleral junction. Measurements were taken at the subfoveal choroid and at $500 \mu \mathrm{m}$ intervals from the fovea: temporal 500 $\mu \mathrm{m}$ (T500), $1000 \mu \mathrm{m}$ (TI000), $1500 \mu \mathrm{m}$ (TI500) and nasal $500 \mu \mathrm{m}$ (N500), $1000 \mu \mathrm{m}$ (NI000) and I500 $\mu \mathrm{m}$ (NI500). 
Caucasian. A sample characterization of the patients and a characterization of tomographic and pachymetric indices (Kmax, Kmed, K2, PachyMin, D) are listed in Table 2.

Of the 80 patients, 51 (64\%) had a positive history of atopy. AR was present in 41 patients, AA in 13 patients and 14 patients had AD. The distribution of atopic conditions is presented in Table 3.

Patients were also classified based on the frequency of eye rubbing. A total of 14 patients (18\%) reported no eye rubbing habits, 27 (34\%) said they rarely rub their eyes, 27 (34\%) said they sometimes rub their eyes and $12(15 \%)$ said they often rub their eyes, making a total of $66(83 \%)$ with eye rubbing habits.

Regarding $\mathrm{KC}$ classification, using Pentacam HR Topographical Keratoconus Classification (TKC), 8 eyes were classified in class $0.5,4$ eyes in class 1,4 eyes in class $1.5,13$ eyes in class 2,13 eyes in class $2.5,28$ eyes in class 3 and 10 eyes in class 3.5 .

Atopic and non-atopic groups are comparable regarding frequency variables and tomographic indices (Table 4). On the other hand, atopic group was significantly more eye rubbing than the non-atopic group ( $p=0.003$ ).

Mean CT was consistently higher in the atopic group $(n=51)$ in all the evaluated points (Figure 2), although without reaching statistically significant levels (mean subfoveal CT $391.53 \pm 108.08 \mu \mathrm{m}$ vs $351.17 \pm 85.60 \mu \mathrm{m}$ in atopic group and non-atopic group, respectively $(p=0.088)$ ).

Each subgroup of atopic patients (AR, AA or AD) was also compared with the non-atopic group. All the subgroups have higher non-significant mean CT than the non-atopic patients. Subfoveal mean CT values obtained were 381.22 $\pm 112.52 \mu \mathrm{m}$ in the AR subgroup $(p=0.230) ; 376.23 \pm 75.99$ $\mu \mathrm{m}$ in the AA subgroup $(p=0.370)$ and $405.55 \pm 106.50 \mu \mathrm{m}$ in $\mathrm{AD}$ subgroup $(p=0.060)$.
Table 3 Distribution of Atopic Conditions in Keratoconus Patients

\begin{tabular}{|l|c|c|}
\hline & N & $\%$ \\
\hline A Rhinitis & 25 & $31 \%$ \\
Asthma & 2 & $3 \%$ \\
A Dermatitis & 7 & $9 \%$ \\
A Rhinitis + Asthma & 6 & $8 \%$ \\
A Rhinitis + A Dermatitis & 6 & $8 \%$ \\
Asthma + A Dermatitis & 1 & $1 \%$ \\
A Rhinitis + Asthma + A Dermatitis & 4 & $5 \%$ \\
None & 29 & $36 \%$ \\
\hline
\end{tabular}

Abbreviations: $n$, number of patients; \%, percentage.

A multivariable analysis was performed adjusting CT for sex, age, spherical equivalent, history of medication and atopy, as these factors could be influencers of choroidal profile. In the adjusted model (Table 5), subfoveal CT in the atopic group is on average $55.14 \mu \mathrm{m}$ thicker than in the nonatopic group, reaching statistical significance $(p=0.043)$ ).

Analyzing AR, AA and AD as atopy subgroups in the adjusted model, we found that patients with Atopic Dermatitis had a significantly thicker choroid compared with non-atopic patients (mean adjusted subfoveal CT increase of $62.66 \mu \mathrm{m}(p=0.035))$. Mean adjusted CT of $\mathrm{AR}$ and $\mathrm{AA}$ patients is not different from non-atopic patients ( $p=0.296$ and $p=0.335$, respectively).

Considering eye rubbing habits, the frequent eye rubber group had a significantly thicker choroid compared to non-rubbers $(p=0.004)$ (Table 6).

\section{Discussion}

An association between atopy, eye rubbing, and $\mathrm{KC}$ has been postulated for many years, and there is strong evidence that atopic traits are more common in patients with $\mathrm{KC}$ than in the

Table 2 Summary of the Characteristics of the Patients' Sample and Characterization of Tomographic Indices

\begin{tabular}{|c|c|c|c|}
\hline & Mean (士SD) & Minimum & Maximum \\
\hline Women, $\mathrm{n}$ & $n=19,24 \%$ & & \\
\hline Right eye, $n$ & $\mathrm{n}=68,85 \%$ & & \\
\hline Age, years & $24.50(4.40)$ & 14 & 30 \\
\hline BCVA, decimal & $0.79(0.21)$ & 0.10 & 1.00 \\
\hline Spherical equivalent, diopters & $-2.35(2.27)$ & -8.50 & 1.75 \\
\hline Kmax, diopters & $56.72(7.95)$ & 42.10 & 82.10 \\
\hline $\mathrm{Km}$, diopters & $48.39(5.37)$ & 39.70 & 67.50 \\
\hline K2, diopters & $50.05(5.94)$ & 40.60 & 71.40 \\
\hline PachyMin, um & $457.15(52.81)$ & 262.00 & 555.00 \\
\hline $\mathrm{D}$ & $9,54(5.30)$ & 0.13 & 30.35 \\
\hline
\end{tabular}

Note: Results are expressed as mean \pm SD (standard deviation) for continuous variables and female gender and right eyes expressed as count and percentage. Abbreviations: BCVA, best corrected visual acuity; Kmax, maximum keratometry; Km, mean keratometry; K2, keratometry of the steepest meridian; PachyMin, minimum pachymetry; D, Belin/Ambrósio D-Index. 
Table 4 Characterization of Tomographic Indices in Atopic and Non-Atopic Groups

\begin{tabular}{|l|c|c|c|}
\hline & Atopic & Non-Atopic & $\boldsymbol{p}$ \\
\hline BCVA, decimal & $0.80(0.2 \mathrm{I})$ & $0.77(0,2 \mathrm{I})$ & $p=0.48 \mathrm{I}$ \\
Spherical equivalent, diopters & $-2.43(2.36)$ & $-2.19(2.14)$ & $p=0.650$ \\
Kmax, diopters & $56.97(8.62)$ & $65.28(6.73)$ & $p=0.709$ \\
Km, diopters & $48.67(5.58)$ & $47.88(5.02)$ & $p=0.529$ \\
K2, diopters & $50.37(6.30)$ & $49.48(5.3 \mathrm{I})$ & $p=0.522$ \\
PachyMin, um & $450.86(48.82)$ & $468.21(58.43)$ & $p=0.159$ \\
D & $9.71(2.36)$ & $9.23(5.32)$ & $p=0.704$ \\
\hline
\end{tabular}

Note: Results are expressed as mean \pm SD (standard deviation).

Abbreviations: BCVA, best corrected visual acuity; Kmax, maximum keratometry; Km, mean keratometry; K2, keratometry of the steepest meridian; PachyMin, minimum pachymetry; D, Belin/Ambrósio D-Index.

general population. ${ }^{1,11}$ Although not truly understood, one possible explanation for the relation between atopy and $\mathrm{KC}$ resides in inflammation and eye rubbing habits. Actually, there is growing evidence of inflammatory mechanisms linked to $\mathrm{KC}$ pathophysiology. ${ }^{1,10}$ In addition to local activation of inflammatory pathways, there is increasing evidence that systemic inflammatory changes and systemic oxidative stress may affect the corneal microenvironment in $\mathrm{KC}$, which may explain the linkage between atopy and $\mathrm{KC}^{7,8}$

Choroid, that has previously been implicated in several inflammatory ocular and systemic diseases, was recently shown to be thicker in KC patients. ${ }^{13-15}$ The exact

\section{Choroidal Thickness in Atopic and Non-Atopic Patients}

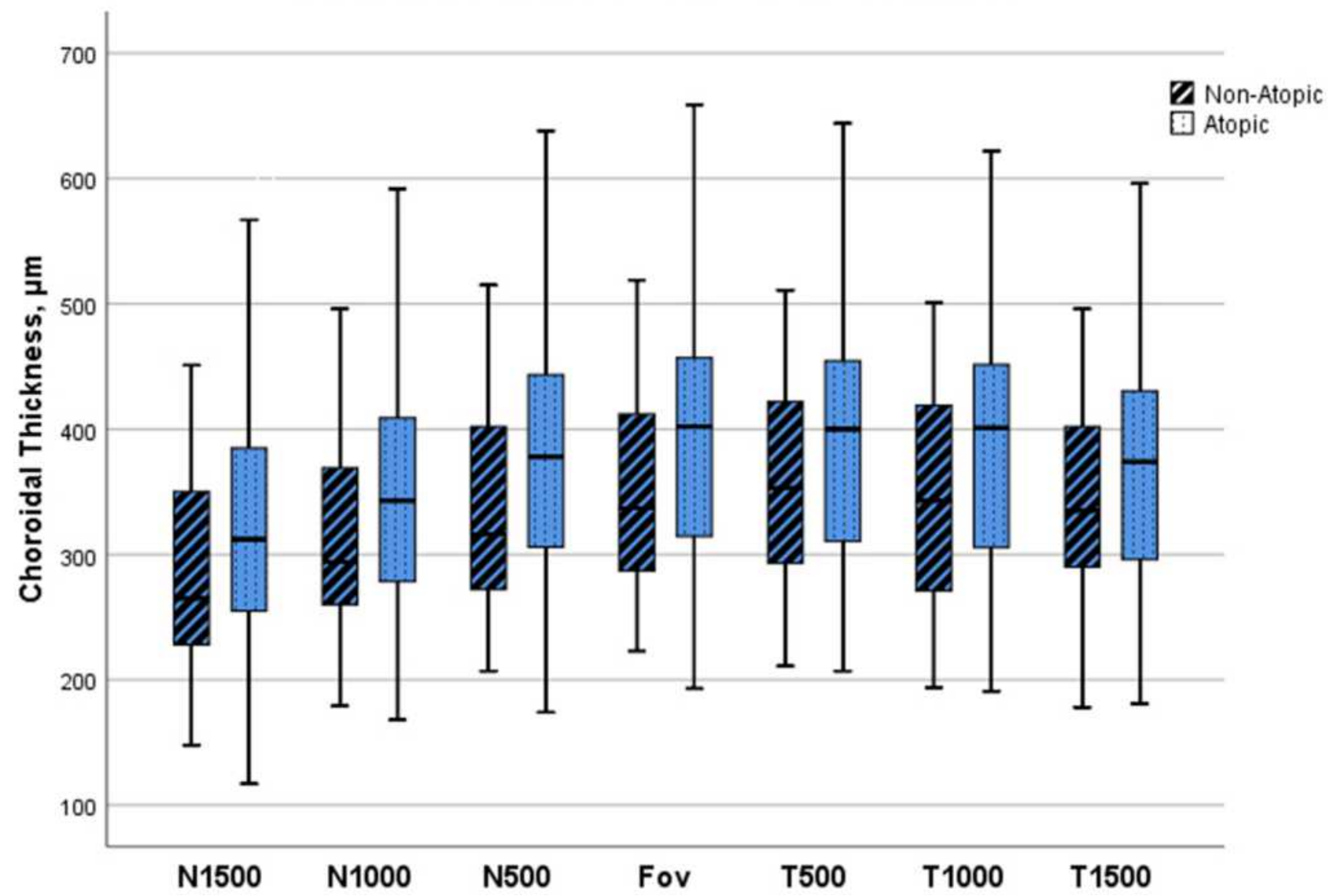

Figure 2 Boxplot results of each choroidal point analyzed in atopic and non-atopic groups. Results are expressed as median ( \pm SD). Measurements undertaken at subfoveal (Fov), temporal $500 \mu \mathrm{m}$ (T500), $1000 \mu \mathrm{m}$ (TI000), $1500 \mu \mathrm{m}$ (TI500), and nasal $500 \mu \mathrm{m}$ (N500), $1000 \mu \mathrm{m}$ (NI000), and I500 $\mu \mathrm{m}$ (NI500). Atopic patients show thicker choroids in every analyzed point, although the differences are not statistically different. 
Table 5 Multivariable Linear Regression Analysis

\begin{tabular}{|l|c|c|c|}
\hline & $\begin{array}{c}\text { B } \\
\text { Coefficient }\end{array}$ & $\begin{array}{c}\text { 95\% Confidence } \\
\text { Interval for } \boldsymbol{\beta}\end{array}$ & p-value \\
\hline Intercept & 356,07 & 219.49 to 492.64 & - \\
\hline Atopy & 55.14 & 1.859 to 108.43 & 0.043 \\
\hline $\begin{array}{l}\text { Allergic } \\
\text { Rhinitis }\end{array}$ & 26.66 & -23.785 to 77.18 & 0.296 \\
\hline $\begin{array}{l}\text { Allergic } \\
\text { Asthma }\end{array}$ & 27.69 & -29.71 to 85.09 & 0.335 \\
\hline $\begin{array}{l}\text { Atopic } \\
\text { Dermatitis }\end{array}$ & 62.66 & 4.79 to 120.52 & 0.035 \\
\hline
\end{tabular}

Notes: Multivariable linear regression analysis with an adjusted model for sex, age, spherical equivalent, history of medication and atopy, including subgroups (allergic rhinitis, allergic asthma and atopic dermatitis). Dependent variable: subfoveal choroidal thickness (CT), $\mu \mathrm{m}$. Adjusted $\mathrm{R}^{2}$ for the model was $2.9 \%$.

Table 6 Subfoveal Choroidal Thickness According to Eye Rubbing Habits

\begin{tabular}{|l|c|c|c|}
\hline Rubbing Habits & Mean & ISD & $\boldsymbol{p}$ \\
\hline None & 337.64 & 111.62 & - \\
Rarely & 379.81 & 104.33 & $p=0.238 *$ \\
Sometimes & 375.48 & 72.61 & $p=0.283 *$ \\
Frequently & 419.33 & 101.84 & $p=0.004 *$ \\
\hline
\end{tabular}

Notes: ${ }^{*}$ p value versus none rubbing habits. Values are expressed in $\mu \mathrm{m}$. Abbreviation: SD, standard deviation.

pathophysiological mechanism resulting in a thicker choroid in $\mathrm{KC}$ patients is not known. However, it raises the possibility of an association between $\mathrm{CT}$ and inflammatory choroidal mechanisms in keratoconic eyes and a possible role for atopy in this mechanism. In this study, we assessed the CT profile of atopic and non-atopic KC patients using SD-OCT technology. To the best of our knowledge, this is the first work analyzing the role of atopy in the choroidal profile of KC patients.

Our results showed a consistent tendency for increased $\mathrm{CT}$ in patients with atopic diseases. Although some results do not reach statistical significance, mean CT is higher in all the evaluated groups of atopic patients when compared to similar $\mathrm{KC}$ patients without known atopy.

It is known that spherical equivalent, age and antiinflammatory drugs could influence CT. Thus, we performed a multivariable analysis adjusted to covariates sex, age, spherical equivalent, history of medication and atopy to access the influence of atopy in the choroidal profile. The use of antihistaminic, non-steroidal anti-inflammatory or corticosteroids drugs was analyzed in the adjusted model.
$\mathrm{KC}$ grade was not considered for adjusting since there is evidence that it is not correlated with $\mathrm{CT}^{13,14}$ After the adjustment, the consistent tendency for a thicker choroid in the atopic patients remained, reaching statistical significance; and the subgroup evaluation revealed that $\mathrm{AD}$ patients have a significantly thicker choroid compared to non-atopic patients. Rhinitis and asthmatic patients had thicker choroids but did not reach the statistical significance.

The highest association shown for $\mathrm{AD}$, but not for the other atopic conditions, may reside in several factors. First, the number of patients included in each subgroup are small, it is possible that with a larger sample the differences observed could be more pronounced. Second, the differences may also reside in the pathogenesis of the disease. ${ }^{20,21} \mathrm{AD}$ pathogenesis is complex and seems to result from a combination of genetic and environmental factors that induce skin barrier dysfunction, cutaneous and systemic immune dysregulation, and skin microbiota dysbiosis. ${ }^{20,21}$ The skin barrier abnormalities appear to be associated with mutations or impaired expression of the filaggrin, ${ }^{20}$ and filaggrin is also expressed in the cornea epithelium ${ }^{22}$ with a major role in the corneal epithelial barrier. ${ }^{22}$ Recent studies showed an altered filaggrin pattern in $\mathrm{KC}$ patients, and revealed association of some mutations in the filaggrin gene with $\mathrm{KC} .^{23}$ These findings may indicate that $\mathrm{KC}$ and $\mathrm{AD}$ share some pathogenic pathways, explaining the highest link found between these two entities. Moreover, AD patients have an altered inflammatory profile, with immune dysregulation and increased type 2 immune cytokines, ${ }^{20}$ which could be related to the increased $\mathrm{CT}$ in these group of patients.

There are only two papers in the literature regarding $\mathrm{CT}$ in atopic conditions. Alper Yenigun et al revealed that choroid was significantly thicker in patients with AR. ${ }^{24}$ These results are in agreement with our findings, although we did not reach a significant difference in the choroidal profile of KC patients with and without AR. On the other hand, Murat Gunay et al found no differences in the subfoveal choroid thickness between asthmatic children treated with corticosteroids and non-asthmatic children. ${ }^{25}$ It is difficult to understand the effects of asthma per se on CT from this work since children were exposed to corticosteroids and we expect anti-inflammatory drugs to reduce the effects of the disease.

Another important finding among our results is that patients with eye rubbing habits have thicker choroids, and, particularly, $\mathrm{KC}$ patients who frequently rub their eyes have significantly thicker choroids than the non-rubbers $\mathrm{KC}$ patients. Chronic repetitive and vigorous eye rubbing is 
associated with $\mathrm{KC}$ development and progression. ${ }^{26}$ Some authors advocate the hypothesis that eye rubbing is the driving force behind the development of $\mathrm{KC}$ in patients with atopic syndrome rather than chronic inflammatory processes, defending that mechanical trauma caused by eye rubbing is the main reason for $\mathrm{KC}$ manifestation. ${ }^{12,27}$ On the other hand, both factors may interplay in a vicious cycle, where eye rubbing promotes inflammation which reinforces eye rubbing habits. Several mechanisms have been proposed for corneal remodeling secondary to eye rubbing habits. Ben-Eli et al describe four mechanisms. ${ }^{12}$ The first one, more consensual, is the mechanical rubbing micro-trauma, which leads to loss of corneal rigidity resulting in bulging of the tissue and a cone-shaped structure. The second mechanism proposed is the increase in temperature due to friction. Third, increased distending force on the cornea while the eye is rubbed elevates the intraocular pressure, which then curves and steepens the cornea. The fourth mechanism takes into account the hypothesis that $\mathrm{KC}$ is an inflammatory state that may be accelerated by increased release of inflammatory mediators. Evidence supporting the inflammatory role in $\mathrm{KC}$ pathogenesis is undeniable. An imbalance in the activity of matrix metallopeptidases (MMPs), increased levels of Interleukin (IL)-4, IL-6, IL-10, RANTES, Interferon (IFN) gamma, Tumor Necrosis Factor (TNF) - alpha cytokine and oxidative stress were all shown to be present in KC patients. ${ }^{1,5-7,9,28}$ Furthermore, it is known that eye rubbing induces mast cell degranulation and an increase of neutrophils and macrophages in the substantia propria of conjunctiva. ${ }^{29}$

Actually, overall evidence considers $\mathrm{KC}$ as a multifactorial disease in which several different pathways have a role. ${ }^{2,12}$ The increased $\mathrm{CT}$ in patients who frequently rub their eyes in our results may suggest that inflammation may effectively play a role in this process, besides the mechanical effects. Also, our results showed that atopy is significantly associated with eye rubbing, supporting the idea that inflammation promoted by these diseases may actually be related to increased CT.

Moreover, Gutierrez-Bonet et $\mathrm{al}^{14}$ observed that the increased CT is only found till age 45 , corresponding to the most active phase of KC's progression; and, it is well known that the incidence of atopic diseases is higher in the first decades of life. ${ }^{30}$ Furthermore, the same authors recently studied the exact components of the thicker choroid in $\mathrm{KC}$ and found that vascular region and corrected choroidal percentage of vascularity are also statistically increased in $\mathrm{KC}$ patients when compared with healthy controls. ${ }^{31}$ If these findings are only an association without any role in the $\mathrm{KC}$, or if they are part of a complex pathogenesis process is still unknown. The overall evidence and our results support the idea that inflammatory mechanisms could contribute both to $\mathrm{KC}$ activity and increase of CT, claiming a role for inflammation and potentially for atopy in its pathophysiology.

Some limitations of this work have to be considered. First, this is a cross-sectional study, which clouds the determination of a causal relationship between atopy, altered choroid profile and $\mathrm{KC}$ development. Considering the vascular nature of the choroid, it would be important to understand if the $\mathrm{CT}$ suffers changes with $\mathrm{KC}$ evolution or worsening of atopic symptoms. Moreover, a longitudinal study could control better some possible biases of this single point observation.

Second, the generalizability of the data may not be assured because we just included 80 patients, all attending the same medical tertiary center, which might lead to an overrepresentation of more severe forms of the disease. Furthermore, the number of patients included in each subgroup are different and small, which limit results' interpretation.

The above-mentioned limitations may also explain the lack of statistical significance in some of our results. Indeed, although the consistent tendency for thicker choroids in atopic patients, some of our results did not reach statistical significance. Furthermore, the adjusted $\mathrm{R}^{2}$ of our linear model is low, precluding a higher power of this study. This evidences that several other factors influence CT besides the ones employed in our model. Part of this problem may reside in the sample size. Further studies employing prior sample size and power calculations will allow to obtain more external validity.

Additionally, all the patients included were Caucasian, which may also limit the generalization of our results to other populations. Moreover, although the adjustment for anti-inflammatory medication history, possible effects of non-mentioned systemic medication can still be a limitation of this work.

In conclusion, our study showed that keratoconic eyes of patients with atopy seem to have thicker choroids compared with keratoconic eyes of non-atopic patients. These results suggest that atopy may have a role in the choroidal profile of KC. Although further studies are necessary to prove and help clarify our findings, this constitutes a completely new sight into Choroidal Thickness in Keratoconus.

\section{Disclosure}

The authors have no financial interest to disclose and report no conflicts of interest for this work. 


\section{References}

1. Galvis V, Sherwin T, Tello A, Merayo J, Barrera R, Acera A. Keratoconus: an inflammatory disorder? Eye. 2015;29(7):843-859. doi:10.1038/eye.2015.63

2. Mas Tur V, MacGregor C, Jayaswal R, O'Brart D, Maycock N. A review of keratoconus: diagnosis, pathophysiology, and genetics. Surv Ophthalmol. 2017;62(6):770-783. doi:10.1016/j. survophthal.2017.06.009

3. Ionescu $\mathrm{C}$, Corbu $\mathrm{CG}$, Tanase $\mathrm{C}$, et al. Inflammatory biomarkers profile as microenvironmental expression in keratoconus. Dis Markers. 2016;2016:1243819. doi:10.1155/2016/1243819

4. Shetty R, Ghosh A, Lim RR, et al. Elevated expression of matrix metalloproteinase- 9 and inflammatory cytokines in keratoconus patients is inhibited by cyclosporine A. Invest Ophthalmol Vis Sci. 2015;56(2):738-750. doi:10.1167/iovs.14-14831

5. Lema I, Sobrino T, Durán JA, Brea D, Díez-Feijoo E. Subclinical keratoconus and inflammatory molecules from tears. $\mathrm{Br}$ J Ophthalmol. 2009;93(6):820-824. doi:10.1136/bjo.2008.144253

6. Sorkhabi R, Ghorbanihaghjo A, Taheri N, Ahoor MH. Tear film inflammatory mediators in patients with keratoconus. Int Ophthalmol. 2015;35(4):467-472. doi:10.1007/s10792-0149971-3

7. Toprak I, Kucukatay V, Yildirim C, Kilic-Toprak E, Kilic-Erkek O. Increased systemic oxidative stress in patients with keratoconus. Eye. 2014;28(3):285-289.

8. Sobrino T, Regueiro U, Malfeito M, et al. Higher expression of toll-like receptors 2 and 4 in blood cells of keratoconus patiens. Sci Rep. 2017;7(1):12975. doi:10.1038/s41598-017-13525-7

9. Wisse RP, Kuiper JJ, Gans R, Imhof S, Radstake TR, Van der Lelij A. Cytokine expression in keratoconus and its corneal microenvironment: a systematic review. Ocul Surf. 2015;13(4):272-283. doi:10.1016/j.jtos.2015.04.006

10. Ferrari G, Rama P. The keratoconus enigma: a pathogenesis review. Ocul Surf. 2020. doi:10.1016/j.jtos.2020.03.006

11. Hashemi H, Heydarian S, Hooshmand E, et al. The prevalence and risk factors for keratoconus: a systematic review and meta-analysis. Cornea. 2020;39(2):263-270. doi:10.1097/ICO.0000000000002150

12. Ben-Eli H, Erdinest N, Solomon A. Pathogenesis and complications of chronic eye rubbing in ocular allergy. Curr Opin Allergy Clin Immunol. 2019;19(5):526-534. doi:10.1097/ACI.0000000000000571

13. Pinheiro-Costa J, Viana Pinto J, Perestrelo S, et al. Increased choroidal thickness in keratoconus patients: perspectives in the disease pathophysiology. J Ophthalmol. 2019;2019:2453931. doi:10.1155/ 2019/2453931

14. Gutierrez-Bonet R, Ruiz-Medrano J, Pena-Garcia P, et al. Macular choroidal thickening in keratoconus patients: Swept-Source Optical Coherence Tomography Study. Transl Vis Sci Technol. 2018;7(3):15. doi:10.1167/tvst.7.3.15

15. Bilgin B, Karadag AS. Choroidal thickness in keratoconus. Int Ophthalmol. 2020;40(1):135-140. doi:10.1007/s10792-01901156-y

16. Steiner M, Esteban-Ortega MDM, Munoz-Fernandez S. Choroidal and retinal thickness in systemic autoimmune and inflammatory diseases: a review. Surv Ophthalmol. 2019;64(6):757-769. doi:10.1016/ j.survophthal.2019.04.007
17. Fong AH, Li KK, Wong D. Choroidal evaluation using enhanced depth imaging spectral-domain optical coherence tomography in Vogt-Koyanagi-Harada disease. Retina. 2011;31(3):502-509. doi:10.1097/IAE.0b013e3182083beb

18. Jap A, Chee SP. The role of enhanced depth imaging optical coherence tomography in chronic Vogt-Koyanagi-Harada disease. $\mathrm{Br}$ $J$ Ophthalmol. 2017;101(2):186-189. doi:10.1136/bjophthalmol2015-308091

19. Ferreira CS, Beato J, Falcão MS, Brandão E, Falcão-Reis F, Carneiro ÂM. Choroidal thickness in multisystemic autoimmune diseases without ophthalmologic manifestations. Retina. 2017;37 (3):529-535. doi:10.1097/IAE.0000000000001193

20. Torres T, Ferreira EO, Goncalo M, Mendes-Bastos P, Selores M, Filipe P. Update on atopic dermatitis. Acta Méd Port. 2019;32 (9):606-613. doi:10.20344/amp.11963

21. Kim J, Kim BE, Leung DYM. Pathophysiology of atopic dermatitis: clinical implications. Allergy Asthma Proc. 2019;40(2):84-92. doi:10.2500/aap.2019.40.4202

22. Tong L, Corrales RM, Chen Z, et al. Expression and regulation of cornified envelope proteins in human corneal epithelium. Invest Ophthalmol Vis Sci. 2006;47(5):1938-1946. doi:10.1167/iovs.05-1129

23. Droitcourt C, Touboul D, Ged C, et al. A prospective study of filaggrin null mutations in keratoconus patients with or without atopic disorders. Dermatology. 2011;222(4):336-341. doi:10.1159/ 000328408

24. Yenigun A, Elbay A, Dogan R, Ozturan O, Ozdemir MH. The effect of allergic rhinitis with positive skin prick test on choroidal thickness. Eur Arch Otorhinolaryngol. 2017;274(6):2477-2481. doi:10.1007/ s00405-017-4513-z

25. Gunay M, Dogru M, Celik G, Gunay BO. Swept-source optical coherence tomography analysis in asthmatic children under inhaled corticosteroid therapy. Cutan Ocul Toxicol. 2019;38(2):131-135. doi:10.1080/15569527.2018.1539009

26. Najmi H, Mobarki Y, Mania K, et al. The correlation between keratoconus and eye rubbing: a review. Int J Ophthalmol. 2019;12 (11):1775-1781. doi:10.18240/ijo.2019.11.17

27. Bawazeer AM, Hodge WG, Lorimer B. Atopy and keratoconus: a multivariate analysis. $\mathrm{Br} J$ Ophthalmol. 2000;84(8):834-836. doi:10.1136/bjo.84.8.834

28. Di Martino E, Ali M, Inglehearn CF. Matrix metalloproteinases in keratoconus - too much of a good thing? Exp Eye Res. 2019;182:137-143. doi:10.1016/j.exer.2019.03.016

29. Greiner JV, Peace DG, Baird RS, Allansmith MR. Effects of eye rubbing on the conjunctiva as a model of ocular inflammation. $\mathrm{Am}$ $J$ Ophthalmol. 1985;100(1):45-50. doi:10.1016/S0002-9394(14) 74981-5

30. Zheng T, Yu J, Oh MH, Zhu Z. The atopic march: progression from atopic dermatitis to allergic rhinitis and asthma. Allergy Asthma Immunol Res. 2011;3(2):67-73. doi:10.4168/aair.2011.3.2.67

31. Gutierrez-Bonet R, Ruiz-Medrano J, Biarnés M, et al. Analysis of choroidal vascularity index in keratoconus patients using swept-source optical coherence tomography-based binarization techniques. J Ophthalmol. 2020;2020:1682463. doi:10.1155/2020/ 1682463 


\section{Publish your work in this journal}

Clinical Ophthalmology is an international, peer-reviewed journal covering all subspecialties within ophthalmology. Key topics include Optometry; Visual science; Pharmacology and drug therapy in eye diseases; Basic Sciences; Primary and Secondary eye care; Patient Safety and Quality of Care Improvements. This journal is indexed on PubMed

Submit your manuscript here: https://www.dovepress.com/clinical-ophthalmology-journal
Central and CAS, and is the official journal of The Society of Clinical Ophthalmology (SCO). The manuscript management system is completely online and includes a very quick and fair peer-review system, which is all easy to use. Visit http://www.dovepress.com/ testimonials.php to read real quotes from published authors. 